Service social

\title{
État des connaissances à l'origine de l'utilisation du logiciel Vie familiale et impact de la maladie : attitudes et motivation de la clientèle et préoccupation des professionnels
}

\author{
Martin Poulin, André Beaudoin et Françoise Turgeon-Krawczuk
}

Volume 41, numéro 3, 1992

Intervenir en contexte d'autorité

URI : https://id.erudit.org/iderudit/706587ar

DOI : https://doi.org/10.7202/706587ar

Aller au sommaire du numéro

Éditeur(s)

École de service social de l'Université Laval

ISSN

1708-1734 (numérique)

Découvrir la revue

Citer cette note

Poulin, M., Beaudoin, A. \& Turgeon-Krawczuk, F. (1992). État des connaissances à l'origine de l'utilisation du logiciel Vie familiale et impact de la maladie : attitudes et motivation de la clientèle et préoccupation des professionnels. Service social, 41(3), 105-123. https://doi.org/10.7202/706587ar
Résumé de l'article

L'utilisation de l'ordinateur dans l'intervention en service social se répand de plus en plus même si elle est encore assez peu fréquente dans notre milieu. Un exemple de ce type d'application correspond au développement et à l'utilisation du logiciel Vie familiale et impact de la maladie auprès des parents d'enfants atteints de fibrose kystique à l'hôpital Sainte-Justine à Montréal et au Centre hospitalier de l'Université Laval (CHUL) à Québec entre 1989 et 1991.

En vue de l'utilisation directe et interactive de ce logiciel avec les parents, il a été nécessaire de déterminer quelles devaient être les conditions à respecter pour assurer le succès de l'expérimentation. On se devait donc de faire le point de l'état des connaissances sur les résistances et les attentes de la clientèle à l'égard de la nouvelle technologie, sur les préoccupations des professionnels envers les applications cliniques de l'informatique et sur la motivation des utilisateurs à se servir des ordinateurs sur une base longitudinale. Les résultats de cette exploration sont communiqués dans cet article avant que soient présentés, en dernier lieu, le contenu du logiciel et son mode d'utilisation. 


\title{
État des connaissances à l'origine de l'utilisation du logicie/ Vie familiale et impact de la maladie : attitudes et motivation de la clientèle et préoccupations des professionnels
}

\author{
Martin POULIN \\ Professeur, École de service social \\ Université Laval \\ ANDRÉ BEAUDOIN \\ Professeur, École de service social \\ Université Laval
}

FRANÇOISE TURGEON-KRAWCZUK Chercheuse, hôpital Rivière-des-Prairies Montréal

\section{INTRODUCTION}

L'utilisation de l'ordinateur dans l'intervention en service social se répand de plus en plus, bien qu'elle soit encore assez peu fréquente dans notre milieu. Un exemple de ce type d'application correspond au développement et à l'utilisation du logiciel Vie familiale et impact de la maladie auprès des parents d'enfants atteints de fibrose kystique à l'hôpital Sainte-Justine à Montréal et au Centre hospitalier de l'Université Laval (CHUL) à Québec, entre 1989 et 1991.

Au moment de la mise en place d'un tel logiciel, il a été nécessaire de s'assurer qu'il soit accessible, facile à employer et acceptable pour les utilisateurs 
potentiels. Avant de s'engager dans cette voie, il a fallu faire le point sur l'état des connaissances relatives aux résistances et aux attentes de la clientèle à l'égard de la nouvelle technologie, sur les préoccupations des professionnels envers les applications cliniques de l'informatique et sur la motivation des utilisateurs à se servir des ordinateurs sur une base longitudinale. C'est des résultats de cette exploration qu'il sera question dans les trois premières parties de cet article, avant que soient présentés, en dernier lieu, le contenu du logiciel et son mode d'utilisation.

\section{LES ATTITUDES DE RÉSISTANCE ET LES ATTENTES DE LA CLIENTÈLE}

Les nombreuses études portant sur les réactions de la clientèle à l'utilisation des ordinateurs constatent, de façon générale, que ces réactions sont positives.

Une des études classiques est celle de Skinner et Allen (1983), qui comparent les renseignements sur l'usage d'alcool, de drogue et de tabac obtenus par trois modes d'évaluation : I'entrevue informatisée, l'entrevue face-àface et le questionnaire crayon-papier. La population à l'étude comprend 150 personnes, présentant des problèmes d'alcool ou de drogue, qui sont distribuées au hasard dans les trois groupes d'évaluation. De façon générale, les répondants trouvent l'entrevue informatisée plus intéressante et moins stressante que l'entrevue face-à-face ou que le questionnaire crayon-papier. Cette réponse suggère une acceptation favorable de l'ordinateur.

Skinner et Allen analysent ces résultats en relation avec sept variables : âge, sexe, scolarité et quatre autres caractéristiques concernant les ressources intellectuelles et l'état affectif du répondant, mesurées au préalable par des tests fiables et valides.

L'entrevue informatisée plaît davantage à ceux qui ont une bonne performance visuo-motrice ; elle plaît moins aux personnes très scolarisées qui ont tendance à intellectualiser et à nier les problèmes. L'entrevue face-à-face est préférée par les femmes et par les répondants qui font de la négation; elle est moins bien cotée par ceux qui sont anxieux et dont le niveau d'anxiété peut être exacerbé par le contact interpersonnel. Le questionnaire crayonpapier est le préféré des personnes plus âgées, plus défensives et qui ont de meilleures habiletés verbales.

De nombreux auteurs se sont penchés sur le thème de l'alphabétisation informatique : les personnes qui ne comprennent pas le processus d'utilisation des logiciels, ou leur raison d'être, ou qui ressentent une anxiété excessive devant la nouvelle technologie sont désavantagées par rapport à l'utilisation de l'informatique (Sampson, 1983a ; Johnson et al., 1981 ; Merrell, 1985 ; Hofer, 1985). 
Merrell (1985) souligne que la mesure des aptitudes ou des valeurs individuelles au moyen des tests informatisés peut être biaisée par la compétence plus ou moins grande des personnes à utiliser la machine. Ceux qui ont des connaissances informatiques limitées et ceux que l'ordinateur rend très anxieux peuvent invalider les résultats de leurs tests. Sampson (1983a) abonde dans le même sens. Selon lui, un problème important des applications cliniques de l'informatique est lié aux facteurs humains : la personne peut mal interpréter les instructions d'utilisation ou elle peut éprouver des difficultés à lire et à comprendre les items présentés à l'écran. Elle peut aussi enfoncer la mauvaise touche par erreur et augmenter ainsi son anxiété initiale par crainte d'avoir abîmé quelque chose dans l'ordinateur. D'autres auteurs (Lushene et al., 1974 ; Hedl et al., 1973) rapportent que les tests psychométriques augmentent de façon significative le niveau d'anxiété du client dans la période qui précède immédiatement l'administration du test. Ces diverses études qui datent de nombreuses années se basent sur les dires de la clientèle et n'utilisent aucune mesure directe du fonctionnement physiologique.

La recherche de Hart (1985) cherche à combler ces lacunes. Elle vise à examiner les effets de l'administration d'un test informatisé (MMPI) en relation avec le temps nécessaire pour le remplir, la satisfaction, l'impact et le stress tel que décrit par la clientèle en comparaison avec le stress vérifié par un moniteur physiologique. Les 30 sujets volontaires, répartis au hasard dans trois groupes différents, ont tous un nombre égal d'électrodes fixées au front et aux épaules pendant les trente premières minutes de l'expérience. On leur demande ensuite de remplir une échelle d'évaluation de l'anxiété et un questionnaire d'évaluation de la satisfaction. Les résultats de cette recherche $n^{\prime}$ indiquent aucune différence significative entre les trois groupes, ni dans la satisfaction, ni dans le stress tel que rapporté par la clientèle ou tel que vérifié par le moniteur physiologique. Par contre, les tests informatisés sont remplis plus rapidement et avec moins de réserve, c'est-à-dire avec une plus grande sincérité. Bien que les résultats de cette recherche soient intéressants, ils ne sont pas concluants en raison du petit nombre de sujets participants.

De leur côté, Logie et al. (1976) ont cherché à déceler le taux d'erreurs qui se glissent dans un questionnaire médical informatisé et rempli par la clientèle. Ils s'adressent à 120 patients divisés en deux groupes dirigés par deux médecins et dont les corrections et les commentaires sont vérifiés par un expert. Les résultats démontrent que les erreurs sont très peu fréquentes : une sur 221 questions. Trente-huit pour cent des erreurs sont dues au fait que le patient n'a pas compris la question. Les auteurs soulignent l'importance de pouvoir s'appuyer sur une technologie qui enregistre de façon exacte et fiable les informations fournies par la clientèle.

Les problèmes reliés à l'entrée des données sont aussi commentés par Johnson et al. (1981). Pour ces auteurs, la première possibilité d'erreur est due 
à l'incompréhension des directives données à l'utilisateur. Les solutions à ce problème sont diverses et controversées. Certains (Greist et Klein, 1980), suggèrent de donner des instructions très explicites et d'en vérifier la compréhension. D'autres (Johnson et Williams, 1980) trouvent que cette approche ralentit et irrite les utilisateurs ; ils préfèrent les directives simples et l'appel au superviseur en cas de besoin. Lushene et al. (1976) sont d'avis que la meilleure solution est de faire un essai avec le répondant avant de le laisser utiliser le logiciel.

Hofer et al. (1985) remarquent que les avantages de la technologie ne sont pas distribués également dans la société ; un nouveau biais culturel se manifeste en faveur des personnes qui ont l'habitude de travailler avec les ordinateurs. Ces auteurs établissent une corrélation entre le peu de connaissance de l'informatique et l'âge, le sexe, l'ethnie, le statut socio-économique. Ils admettent que leurs assertions ne sont pas prouvées et que des recherches plus approfondies sont nécessaires dans ce domaine. Ils soulignent le besoin $d^{\prime}$ 'une liste d'indicateurs de problèmes possibles avec l'informatique, qui permettrait de les prévoir et d'y remédier. Entre-temps, ils recommandent la formation des non-initiés et la pratique avec l'ordinateur, aussi longtemps que c'est nécessaire, pour que ceux-ci puissent surmonter leur anxiété et se sentir à l'aise de façon à pouvoir se concentrer sur le contenu du programme.

De son côté, le professionnel peut avoir à intervenir pour réduire au minimum les conséquences d'une utilisation fautive de l'ordinateur par la clientèle ou pour diminuer ses résistances. Selon Sampson (1983b), les utilisateurs montrent une préférence pour l'intervention structurée du clinicien en ce qui regarde la mise en œuvre et le suivi de l'expérience avec l'informatique. Le rôle du professionnel serait donc de déterminer si le client est apte émotivement, physiquement et intellectuellement à utiliser l'ordinateur ; il expliquerait aussi au client le but et les modalités de l'application informatique pour s'assurer de la conformité des attentes du client avec les résultats de l'expérience technologique tels qu'anticipés ; il assurerait ensuite l'accompagnement dans l'utilisation de l'informatique.

Ellis et Raines (1982) décrivent un cheminement différent. Les volontaires qui utilisent leurs programmes éducatifs informatisés sont placés dans trois groupes distincts. Les répondants du premier groupe (assistés) dictent leurs réponses à un aide qui les introduit lui-même dans l'ordinateur ; l'autre groupe interagit directement avec l'ordinateur ; ces personnes peuvent demander de l'aide au besoin (supervisés). Le troisième groupe de personnes utilise l'ordinateur de façon autonome (non supervisés). Les auteurs cherchent à mesurer le rôle de l'environnement en relation avec l'utilité des programmes telle qu'elle est évaluée par la clientèle. Ils concluent que les applications cliniques sont peut-être plus utiles au client qui connaît peu la technologie lorsqu'une aide lui est apportée en cours d'expérience. 
Plusieurs auteurs sont d'opinion que l'accompagnement peut se faire par un non-professionnel, si celui-ci a un bon contact avec la clientèle, s'il est disponible et s'il connaît bien le fonctionnement de l'ordinateur (Space, 1981 ; Ellis et Raines, 1982 ; Merrell, 1985 ; Selmi et al., 1982).

On trouve fréquemment dans la littérature sur le sujet des références aux travaux de recherche de Lucas (1977). Bien qu'on ait déjà demandé l'opinion des utilisateurs dans le cadre de très nombreuses études, personne avant Lucas n'avait tenté de mesurer objectivement leurs attitudes dans ce domaine. Pour évaluer les techniques de collecte automatique des données médicales, Lucas a conçu et validé deux échelles de mesure des attitudes des patients relativement à l'interrogation par ordinateur.

Critiquant la méthode qui consiste à interroger directement la population à l'étude au moyen d'un questionnaire, Lucas en souligne les limites : les personnes expriment rarement une opinion négative, répondant plutôt de façon à plaire au chercheur ; I'interprétation des données ainsi obtenues est souvent biaisée. De plus, il n'existe pas d'analyse de corrélation entre les items permettant de combiner les réponses à chaque question dans un score global d'attitudes.

S'inspirant des recherches de Thurstone et al. (1929), de Franklin et al. (1967) et de Hulka et al. (1970), Lucas a donc construit et utilisé une première échelle comprenant 22 items évalués selon une échelle de type Likert en six points, pour estimer les attitudes favorables ou défavorables à l'ordinateur et pour vérifier la contribution des variables d'analyse comme l'âge, le sexe et la scolarité. Il présente son étude de validation de façon très détaillée : choix des items formulés de façon positive et négative, analyse de la structure unifactorielle, test de consistance interne (coefficient alpha de Cronbach de 0,90), analyse de corrélation (Pearson) inter-items et item-total, fiabilité testretest.

Pour conclure sa démarche, Lucas a construit une seconde échelle (différentielle sémantique) dérivée principalement des travaux de Heise (1965, 1969 et 1970) et des études d'analyse factorielles d'Osgood et al. (1957) pour comparer les attitudes des patients envers " l'entrevue médicale avec un ordinateur », "l'entrevue médicale avec un médecin » et " l'entrevue médicale idéale ». Cette échelle fait l'objet d'une présentation tout aussi rigoureuse que la première : fidélité test-retest $(0,83$ à 0,91$)$, analyse factorielle utilisant le coefficient de Kendall avec des résultats satisfaisants. Lucas poursuit son processus de validation par l'étude de la matrice de corrélation entre les items des deux échelles $(r=0,70)$, venant à la conclusion qu'elles mesurent essentiellement le même phénomène, c'est-à-dire l'attitude à l'égard de I'interrogation par ordinateur.

Pour vérifier ces qualités psychométriques, Lucas soumet les deux échelles et un bref questionnaire sur l'âge, le sexe et la scolarité à 75 patients 
fréquentant une clinique médicale spécialisée. Ces mesures, qui leur sont remises immédiatement après l'interrogation par ordinateur, doivent être remplies à domicile et retournées de façon anonyme par la poste ; Lucas obtient un taux de réponse de $89 \%$. Parmi les 67 patients qui ont répondu, $82 \%$ ont une attitude favorable à l'entrevue par ordinateur ; $49 \%$ ont une attitude plus favorable envers l'entrevue médicale par ordinateur qu'envers l'entrevue médicale avec un médecin. Par ailleurs, les hommes ont des attitudes plus favorables que les femmes, les jeunes sont plus positifs que les personnes plus âgées et les travailleurs manuels montrent une plus grande préférence pour l'ordinateur que les personnes ayant d'autres occupations.

Les recherches sur les attitudes de résistance et d'attente de la clientèle à l'égard de l'utilisation des ordinateurs, fort nombreuses il y a une dizaine d'années, ont beaucoup diminué depuis. Les revues de littérature récentes (Hedlund, 1988 ; Mathisen, 1988 ; Erdman et Foster, 1986 ; Hudson et al., 1988 ; Nurius, 1990) démontrent que l'acceptation de l'informatique par la clientèle est maintenant bien documentée.

\section{LES PRÉOCCUPATIONS DES PROFESSIONNELS RELATIVEMENT À L'UTILISATION DE L'INFORMATIQUE PAR LA CLIENTÈLE}

Dès le moment où les innovations technologiques ont fait participer directement la clientèle par le moyen d'échanges interactifs avec l'ordinateur, les professionnels de la santé mentale ont exprimé leurs inquiétudes devant le changement. On peut regrouper leurs préoccupations autour de trois thèmes principaux qui reviennent souvent dans la littérature et dans les questions de recherche concernant les réactions de la clientèle.

\section{La déshumanisation}

Les entrevues et les tests informatisés dépersonnalisent le client : celui-ci se sent l'objet de manipulations électroniques et mécaniques qui nuisent à la relation bénéficiaire-clinicien et qui augmentent le sentiment d'isolement de la personne dans un univers de plus en plus envahi par la technologie (Space, 1981). De nombreux auteurs (Colby, 1980 ; Erdman et al., 1981 ; Selmi et al., 1982 ; Sampson, 1983) citent Weizenbaum qui s'oppose fortement, pour des raisons d'éthique, à ce que le langage informatique exprime des sentiments humains comme la compréhension et le soutien. Pour lui, l'introduction de I'informatique dans le processus thérapeutique est une fraude (Erdman et Foster, 1986). Son opinion est battue en brèche par la plupart des auteurs qui font référence à ses écrits et qui soulignent que la clientèle réagit très favorablement à l'utilisation des ordinateurs. 
La controverse n'est certainement pas terminée. De nombreuses recherches ont tenté d'élucider ce problème. Klinger et al. (1976-1977), dans leur évaluation du programme PAU (Psychiatric Assessment Unit), ont questionné 132 patients pour chercher à cerner leur opinion sur « I'ordinateur déshumanisant ». Les résultats de la recherche démontrent que les patients ne croient pas qu'il s'agisse d'une expérience déshumanisante pour eux : $68 \%$ ont aimé être interrogés par ordinateur ; 57 \% ont préféré les entrevues informatisées aux entrevues cliniques; 46 \% se sont dits plus sincères avec l'ordinateur ; $21 \%$ seulement de la population à l'étude a trouvé les tests informatisés trop impersonnels.

Dans le cadre de sa recherche sur l'évaluation de la relation maritale, Costello (1982) a interrogé 35 femmes : il cherchait à vérifier si ces femmes réagiraient favorablement à l'utilisation de l'ordinateur dans I'administration d'une batterie de dix tests. Toutes les participantes à l'étude ont trouvé que I'ordinateur facilitait leurs réponses aux différents tests, parce que leur contact avec la machine était moins personnel et moins menaçant ; dans une entrevue traditionnelle avec un clinicien, elles se seraient trouvées gênées de répondre, affirment-elles.

Commentant sur le thème de la déshumanisation, Colby (1980) souligne la tendance des gens à l'anthropomorphisme, c'est-à-dire à traiter comme un être humain toute entité qui peut s'engager dans une communication symbolique. Il ne croit pas que l'ordinateur puisse avoir un effet délétère sur le patient. D'après lui, la machine peut éliminer les émotions humaines négatives comme la colère, I'ennui, une expression faciale méprisante ; en contrepartie, l'ordinateur est toujours poli, disponible en tout temps; il ne porte pas de jugement moral, il n'a pas un statut social supérieur ; il ne fait pas d'avance sexuelle au patient ; il peut lui remettre une copie de son entrevue.

En conclusion de sa recherche descriptive sur les applications technologiques nouvelles, Stout (1981) signale le besoin d'études approfondies sur le comportement humain dans une situation d'entrevues et de tests informatisés, dans le but de développer un système plus efficace. L'ordinateur peut permettre une approche plus active, plus « personnelle » que celle qui consiste à recueillir de l'information. Des programmes vraiment novateurs pourraient utiliser davantage sa capacité d'observer et d'intervenir, simulant mieux l'entrevue clinique qu'il ne peut remplacer que partiellement.

\section{La confidentialité}

Cette question d'éthique est très souvent mentionnée dans la littérature et les auteurs reconnaissent le bien-fondé d'une telle préoccupation (Space, 1981 ; Mezzich et al., 1982 ; Greist et Klein, 1980 ; Sampson, 1983 ; Merrell, 1985 ; Erdman et al., 1985, 1986). La résistance des cliniciens à l'informatique y est 
souvent reliée (Mezzich et al., 1982 ; Merrell, 1985 ; Greist et Klein, 1981). Les suggestions pour contrôler l'accès à l'information confidentielle incluent I'utilisation de mots de passe, les cotes d'identification des dossiers, les différents niveaux d'accès aux banques de données. Greist et Klein (1981) affirment qu'il est beaucoup plus difficile d'accéder illégalement à l'information contenue dans une banque de données bien gardée que d'obtenir frauduleusement un rapport traditionnel. Dans le cas où un micro-ordinateur est utilisé, Merrell (1985) recommande de conserver les disquettes dans un endroit sûr, de les identifier par un code et d'en effacer le contenu dès qu'elles ne sont plus utiles.

Par ailleurs, la clientèle n'exprime pas les mêmes réticences que les cliniciens à l'égard de la confidentialité, bien au contraire. Les revues de la littérature faites par Erdman et al. (1985), Hedlund et al. (1985), Skinner et Allen (1983), Greist et Klein (1981) soulignent à l'unanimité que les clients révèlent des renseignements confidentiels beaucoup plus facilement à l'ordinateur qu'au clinicien. De très nombreuses recherches tant en médecine qu'en santé mentale appuient leurs propos. Greist et Klein (1980) ont comparé les deux méthodes de collecte d'informations auprès de groupes d'étudiants présentant des problématiques qui risquaient de susciter bien des réticences : l'abus de drogue et d'alcool et la dysfonction sexuelle. Leur recherche sur les problèmes sexuels d'une population sélectionnée au hasard est particulièrement significative : tous les répondants, les femmes surtout, ont révélé beaucoup plus de difficultés sexuelles à l'ordinateur qu'au psychiatre. Cette différence marquée n'était pas évidente dans leurs réponses aux autres questions portant, par exemple, sur la fréquence des exercices physiques. Les répondants ont exprimé l'opinion qu'ils étaient plus à l'aise avec l'ordinateur qui respectait davantage leur intimité.

Erdman et al. (1981) rappellent que les professionnels de la santé mentale doivent chercher à procurer la meilleure qualité de soins possible à la clientèle. Certains patients peuvent trouver que l'ordinateur est un médium trop impersonnel pour être utilisé en relation avec des problèmes émotifs sérieux. D'autres peuvent trouver humiliant de divulguer les détails intimes de leur vie à un étranger, même s'il s'agit d'un professionnel. En dernière analyse, et à la condition qu'on puisse lui offrir un programme thérapeutique informatisé valable, c'est le client qui devrait pouvoir faire son choix entre le clinicien et l'ordinateur.

\section{La standardisation}

Space (1981) résume bien les critiques les plus fréquentes sur ce thème. Les applications technologiques décrivent une personne à un point précis sur un continuum en comparaison avec l'ensemble de la population, mais elles 
ne tiennent pas compte des aspects qui sont propres à cette personne. L'information personnelle qui est la plus importante sur le plan clinique se perd en faveur de l'information abstraite et générale. Ces applications ne permettent pas non plus d'inférences complexes sur la psychodynamique ou sur la motivation qui sous-tend le comportement de la personne, dont la richesse et la complexité se perdent. Les applications sont statiques; elles ne s'adaptent pas au style de réponse de chacun et ne peuvent pas se centrer sur les éléments importants qui sont à la base d'un diagnostic vraiment individualisé. Les rapports informatisés sont stéréotypés et n'apportent rien de vraiment neuf au clinicien.

D’après Space (1981), ces critiques sont formulées par des personnes qui ne connaissent pas le potentiel de l'informatique. Les chercheurs peuvent créer des programmes qui se rapprochent beaucoup des exigences cliniques à la condition de travailler en collaboration étroite avec les professionnels qui sont appelés à les utiliser. Greist et Klein (1981) et Erdman et al. (1985) ont largement approfondi ce thème.

Un effort d'individualisation des programmes a déjà été fait par quelques chercheurs (Sorrell et al., 1982 ; Selmi et al., 1982 ; Carr et Ghosh, 1983), et la clientèle semble y réagir de façon très positive.

Ainsi Carr et Ghosh ont élaboré un logiciel pour évaluer les phobies, les divers comportements des bénéficiaires et les objectifs particuliers de traitement. Le logiciel sélectionne les questions appropriées en fonction des réponses précédentes de l'individu, en s'adressant à lui par son nom ; si la personne fait une erreur (par exemple, si elle répond par un nombre plutôt que par oui ou non), un message explicatif apparaît à l'écran pour lui demander de faire une autre tentative ; si les réponses de l'utilisateur ne sont pas logiques et qu'elles manifestent qu'il n'a pas compris les concepts sous-jacents, I'ordinateur donne des explications ; si la personne ne présente pas de symptômes, le logiciel passe rapidement à une autre série de questions.

Les résultats de la recherche de Carr et Ghosh avec 43 patients dont le diagnostic de phobie est déjà posé par un psychiatre démontrent que 88 \% des sujets à l'étude trouvent l'entrevue par ordinateur aussi acceptable que I'entrevue traditionnelle, surtout à cause de la flexibilité du programme qui leur permet de prendre tout leur temps, les rendant ainsi moins anxieux et moins nerveux. Les auteurs en concluent que si l'ordinateur semble capable d'aider la clientèle à mieux communiquer ses besoins propres au médecin, il doit être exploité avec confiance.

Selmi et al. (1982) apportent un autre exemple d'individualisation d'un programme informatisé (thérapie cognitive de la dépression moyenne ou légère), auquel la clientèle réagit de façon très positive. Cette étude soigneusement contrôlée porte sur une population qui répond aux critères de diagnostic ; ce sont des volontaires qui sont placés au hasard dans l'un des trois groupes de traitement : MORTON (le thérapeute-ordinateur), un clinicien 
traditionnel et un groupe contrôle de traitement sur demande. Comparativement au groupe contrôle, les deux groupes de traitement présentent une amélioration significative sur plusieurs échelles d'évaluation de la dépression. Commentant les résultats de cette recherche, Erdman et al. (1985) émettent l'opinion que de tels programmes informatisés et personnalisés ont un potentiel intéressant d'aide à la clientèle.

Cwikel et Cnaan (1991) sont d'avis que la pratique du service social atteint actuellement une nouvelle étape dans l'utilisation de la technologie informatique, ce qui pose des problèmes d'éthique différents qui doivent être définis et résolus pour permettre une adaptation constructive au changement. Les principes fondamentaux d'autodétermination, d'individualisation du traitement, d'accès égal aux ressources pour tous sont au premier plan des préoccupations d'aujourd'hui en relation avec le développement des applications cliniques de l'informatique en service social. La question de la confidentialité, souvent débattue au cours des vingt dernières années, reste importante mais, comme le soulignent Gandy et Tepperman (1990), elle ne soulève plus la même inquiétude qu'auparavant ; il en est de même de la crainte de la déshumanisation des services par l'ordinateur.

\section{LA MOTIVATION DE LA CLIENTÈLE À UTILISER LA TECHNOLOGIE DE FAÇON RÉPÉTITIVE}

Les références à l'attrait de la nouveauté comme motivation à utiliser l'ordinateur sont assez fréquentes dans la littérature (Ellis et Raines, 1982 ; Erdman et al., 1985). D'après leur expérience, Ellis et Raines remarquent que l'attrait de la clientèle pour les applications informatiques persiste sur une période de plusieurs mois, surtout lorsque les utilisateurs peuvent avoir accès à un certain choix de programmes pour soutenir leur intérêt. Erdman et al. (1985) envisagent la possibilité que la réponse positive de la clientèle à l'ordinateur s'atténue à mesure que l'attrait de la nouveauté diminue : ils se disent pourtant convaincus que la nouveauté n'est pas la variable la plus importante dans I'acceptation par la clientèle. Ils rappellent que certaines entrevues informatisées prennent deux heures, alors qu'une évaluation du comportement comme celle de Angle et al. (1977) prend environ sept heures. Les auteurs sont d'avis que la clientèle peut avoir été attirée de prime abord par la nouveauté, mais que sa réponse positive est motivée par d'autres facteurs. Dans une étude (Angle et al., 1977) de 331 répondants, $15 \%$ seulement ont trouvé l'entrevue trop longue et $80 \%$ étaient prêts à recommencer ; $60 \%$ ont préféré l'ordinateur à l'entrevue personnelle.

En accord avec Lanyon et Johnson (1980), Selmi et al. (1982) croient que les applications technologiques peuvent répondre aux besoins de la clientèle sur le plan de l'efficacité et des coûts. Un bénéficiaire se désintéresse 
vite d'un programme qui n'est pas pertinent, qui le frustre et l'ennuie. Le traitement informatisé mis au point par Selmi comprend huit sessions d'environ une heure chacune, sur une période de six à huit semaines; les utilisateurs n'ont pas de réticence à persister dans cette expérience.

De leur côté, Sorrell et al. (1982) ont conçu un programme visant à favoriser la « compliance » au traitement médical. La « compliance » est la capacité des parents et de l'enfant à répondre adéquatement et de façon soutenue aux ordonnances de l'équipe médicale concernant les soins et les médicaments à donner à l'enfant. Ils cherchent à savoir si, d'une part, la clientèle utilisera son programme informatisé régulièrement sur une période de six semaines et si, d'autre part, le traitement apporté sera efficace sur le plan du renforcement positif des attitudes relatives à la prise de médication prescrite sur une longue période de temps. Pour Sorrell et ses collaborateurs, la motivation est reliée à la perception et à l'acceptation du changement.

\section{LE LOGICIEL «VIE FAMILIALE ET IMPACT DE LA MALADIE » COMME MOYEN D'INTERROGATION INTERACTIVE DANS L'INTERVENTION EN SERVICE SOCIAL}

Les constatations précédentes confirment pour le service social les conclusions de plusieurs auteurs (Steiner, 1981 ; Hofer, 1985 ; Hedlund, 1988 ; Hudson, 1988 ; Nurius, 1990) sur l'acceptation et les dispositions de la clientèle des services sociaux par rapport à l'utilisation de l'ordinateur. C'est dans cette perspective que s'est réalisé le développement du logiciel d'intervention auprès des parents d'enfants atteints de fibrose kystique.

De nombreuses recherches ont démontré que les enfants malades et leur famille présentent un risque élevé de problèmes psychologiques et sociaux (Cadman et al., 1987). Les parents doivent apprendre à vivre avec un stress chronique et ils ont à se mobiliser pour donner des traitements quotidiens tout en maintenant une vie familiale aussi normale que possible. Ils ont aussi à s'adapter rapidement à un milieu hospitalier fort complexe : ils doivent parfois prendre des décisions difficiles concernant les interventions et les traitements médicaux prescrits pour leur enfant. Dans un tel contexte, I'approche biopsychosociale utilisée par les équipes de soins est essentielle à la prise en charge de la maladie. L'intervention du service social, intégrée à cette approche, vise à soutenir les parents, à les aider à comprendre et à interpréter une situation complexe, à augmenter leur compétence à l'aide de stratégies d'adaptation, tout en améliorant leurs relations familiales et sociales.

Le développement du logiciel interactif Vie familiale et impact de la maladie se situe dans le prolongement de la démarche d'informatisation des données à la clinique de fibrose kystique de l'hôpital Sainte-Justine, visant à 
favoriser l'approche biopsychosociale. Le logiciel a été conçu et développé en tant qu'appui à l'intervention du service social. Sa réalisation est le produit d'un travail de collaboration entre l'intervenante de l'équipe' et quelques chercheurs de l'École de psychologie de l'Université Laval' ${ }^{2}$ La programmation a été mise au point avec l'aide d'une firme privée, en 1989, grâce à une subvention du Centre de services sociaux du Montréal métropolitain.

En même temps que se faisaient l'élaboration et la mise au point du logiciel, une demande de subvention pour son évaluation était présentée à la Fondation canadienne de la fibrose kystique. L'obtention de la subvention a permis la réalisation de cette recherche (Poulin et al., 1991) à laquelle s'est greffée une thèse de doctorat (Turgeon-Krawczuk, 1991). Les deux recherches visent des objectifs complémentaires de mesure des effets du logiciel sur les parents d'enfants atteints de fibrose kystique.

\section{Le contenu du logiciel}

Le logiciel Vie familiale et impact de la maladie se compose des quatre tests suivants :

- la mesure de stress psychologique (Lemyre-Tessier, 1986) ;

- I'index de stress parental (Abidin, 1983);

- l'échelle du climat familial (Moos, 1974);

- le questionnaire d'adaptation à la vie de couple (Locke-Wallace, 1959).

Chacun de ces tests peut être fait par les parents directement à l'ordinateur. Immédiatement après, les répondants obtiennent une interprétation d'un certain nombre de résultats jugés plus pertinents pour appuyer l'intervention. Voici un aperçu des quatre tests utilisés et des résultats interprétés à l'écran :

\section{- La mesure du stress psychologique (MSP) de Lemyre-Tessier (1986)}

II s'agit d'un questionnaire auto-administré de 49 items dont le but est de rendre compte de l'état de tension non pathologique vécu par une personne au cours des deux ou trois derniers jours. Le MSP décrit les manifestations physiologiques, comportementales et affectives de l'état de stress selon une expérience phénoménologique commune et reconnue. II s'agit d'une mesure sensible qui rend compte de la réaction de la personne à des stresseurs tels que ceux qui sont vécus par les parents d'enfants

1. F. Turgeon-Krawczuk, hôpital Sainte-Justine.

2. R. Tessier, L. Lemyre, L. Fillion. 
atteints de fibrose kystique. La mesure démontre des qualités de fiabilité et de validité (Lemyre et al., 1990). Dans le logiciel, le score global de ce test est interprété aux parents.

\section{- L'index de stress parental (Abidin, 1983)}

Cet instrument, dont la fiabilité et la validité sont bien établies, comprend 101 items. Il peut être utilisé avec des parents d'enfants de douze ans et moins. Le but de l'instrument est de déterminer ce qui, dans le système parent-enfant, peut être associé à des problèmes ou à des sources d'insatisfaction de la part des parents dans le développement de l'enfant. L'index est multidimensionnel et les facteurs de stress sont organisés autour de deux domaines principaux : celui du parent et celui de l'enfant. Le domaine du parent comprend 54 items, eux-mêmes divisés en sept souséchelles: I'humeur dépressive, l'attachement, les exigences du rôle de parent, le sentiment de compétence, le degré de contact social, la relation avec le conjoint et la santé du parent. Le domaine de l'enfant comprend 47 items regroupés en six sous-échelles : I'adaptabilité, l'acceptabilité, les demandes de l'enfant, l'humeur, I'hyperactivité et le renforcement du parent. Même si le parent doit répondre au test complet, quatre sous-échelles sont l'objet d'interprétation immédiate à l'écran : les demandes de l'enfant, l'humeur dépressive des parents, les exigences du rôle de parent et le degré de contact social.

\section{- L'échelle de climat familial (Moos, 1974)}

Cette échelle comprend 90 items regroupés sous trois vocables : les dimensions relationnelles (cohésion, expression des sentiments et expression des conflits) ; les dimensions de croissance personnelle (I'autonomie, la poursuite de la réussite, l'orientation intellectuelle-culturelle et l'orientation active-récréative ainsi que l'orientation morale religieuse) ; les dimensions de maintien du système familial (l'organisation et le contrôle). Cette mesure a été retenue à cause de ses qualités métriques satisfaisantes et de la pertinence de la forme informatisée du test. Quatre dimensions ont été retenues dans cette échelle pour interprétation immédiate à l'écran à la suite de son utilisation par le répondant : cohésion, expression des sentiments, autonomie et organisation.

\section{- Le questionnaire d'adaptation à la vie de couple (Locke-Wallace, 1959)}

Même si d'autres mesures existent concernant cette dimension, il a été décidé d'utiliser cet instrument déjà connu depuis plus de trente ans parce qu'il est bref et qu'il continue d'être employé dans la pratique. Ses 17 questions mesurent dans leur ensemble le degré d'adaptation qu'on retrouve dans le couple. L'interprétation est faite à l'écran sur l'ensemble de l'échelle. 


\section{Le mode d'utilisation}

Le logiciel permet aux parents de répondre aux tests directement à l'ordinateur, de manière interactive, selon une méthode très simple et suivant les directives qui apparaissent à l'écran.

De façon typique, le parent (père ou mère) est invité à s'asseoir devant le micro-ordinateur et à répondre aux questions proposées. II peut suspendre l'expérience à n'importe quel moment et reprendre à l'intérieur de la même journée. La partie interprétée des résultats des tests est présentée à l'écran sous forme de graphiques accompagnés d'une interprétation rendue immédiatement accessible au parent sans l'intermédiaire d'un clinicien. Les résultats imprimés remis aux parents ne contiennent que les interprétations, sans les graphiques. Les tests peuvent être répétés trois fois, avec des intervalles de quelques mois, ce qui permet à l'utilisateur de comparer ses résultats d'une fois à l'autre.

Le modèle d'ensemble sur lequel se fonde l'expérimentation du logiciel est celui d'une stratégie cognitive de changement autodéterminé. Le logiciel rend l'observation de soi possible pour les parents grâce à la rétroaction immédiate qu'il fournit et à son utilisation répétée qui permet de comparer les résultats sur une longue période. La figure 1 résume ce modèle.

L'interprétation immédiate des résultats faite au parent permet à celuici de savoir quels sont ses points forts et ses points faibles sous les dix dimensions qui font partie de l'interprétation. Il est ainsi en mesure de prêter attention aux points plus faibles et même de consulter au besoin.

Figure 1

\section{Modèle d'expérimentation du logiciel Vie familiale et impact de la maladie}

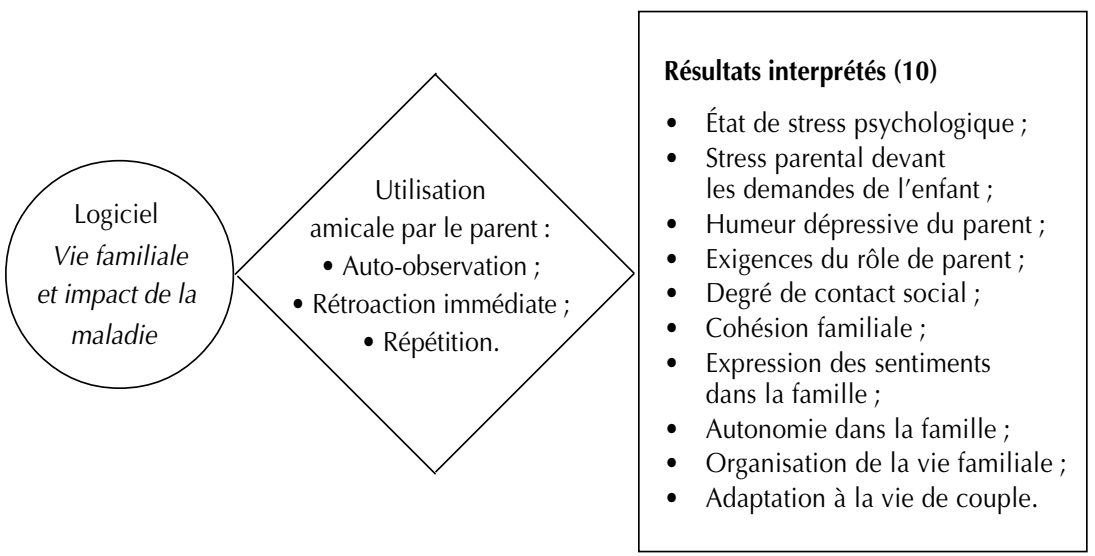


Quatre-vingt-neuf parents d'enfants atteints de fibrose kystique ont accepté de participer à l'expérience de l'utilisation du logiciel Vie familiale et impact de la maladie aux cliniques de fibrose kystique de l'hôpital SainteJustine à Montréal et du CHUL à Québec. Les parents démontraient ainsi des dispositions favorables à l'utilisation de l'informatique comme appui à l'intervention en service social. Malgré le fait que la plupart des répondants $(64,5$ \%) avaient eu peu ou à peu près pas de contact avec l'informatique, les effets de l'utilisation du logiciel se sont révélés évidents sous plusieurs aspects. Ces résultats feront l'objet d'autres publications.

\section{CONCLUSION}

L'utilisation d'un logiciel convivial comme Vie familiale et impact de la maladie constitue un moyen d'intervention acceptable pour les parents d'enfants atteints de fibrose kystique. Ils considèrent qu'ils y trouvent leur bénéfice, et d'autres études devraient sans doute démontrer les mêmes possibilités d'application auprès de parents vivant des situations semblables.

Pourtant, I'utilisation de l'informatique par les travailleurs sociaux comme support à l'intervention directe auprès de la clientèle a été jusqu'à maintenant pratiquement inexistante. En effet, s'il y a des sommes d'argent de plus en plus considérables consacrées à ce domaine dans les différentes organisations de services sociaux, elles servent d'abord à des fins de soutien à la gestion et à la création de banques de données dont l'utilité pour la pratique est tout au plus indirecte. On invoque souvent les dangers de dépersonnalisation et de déshumanisation des clients dans I'utilisation de I'ordinateur. On parle des risques pour la confidentialité et du danger de la standardisation qui empêcherait l'individualisation des situations et des problèmes.

Si ces dangers peuvent être fondés en présence d'une utilisation tous azimuts de l'informatique, les résultats de l'expérience réalisée avec le logiciel Vie familiale et impact de la maladie vont dans le sens contraire. Étant donné la nature du logiciel, son contenu et son mode d'utilisation, la clientèle elle-même réfute en bonne partie les résistances et les objections qu'on trouve chez les intervenants. Au contraire, les constatations de la recherche indiquent clairement qu'on ne peut mettre de côté plus longtemps l'utilisation des nouvelles technologies. Elles sont maintenant disponibles, directement accessibles et contrôlables par les intervenants eux-mêmes, à condition bien entendu que l'utilisation en devienne possible dans le contexte organisationnel du service social et que les modalités d'utilisation soient telles qu'elles respectent des conditions similaires à celles de l'expérience que nous venons de décrire. 


\section{Références bibliographiques}

ABIDIN, R.R. (1983). Parenting Stress Index Manual. Charlottesville, VA : Pediatric Psychology Press.

ANGLE, H.V. et al. (1977). "Computer-aided Interviewing in Comprehensive Behavioral Assessment », Behavior Therapy, $8:$ 47-754.

CADMAN, D., BOYle, M., SzatMari, P. et D. OfFord (1987). "Chronic illness, disability and mental and social well-being : Findings of the Ontario child health study », Pediatrics, vol. 79, n 5 : 805-813.

CARR, A. et A. GHOSH (1983). « Response of phobic patients to direct computer assessment », British Journal of Psychiatry, 142:60-65.

COLBY, K.M. (1980). « Computer psychotherapists », dans J.B. Sidowski, J.H. Johnson et T.A. Williams (eds.), Technology in Mental Health Care Delivery Systems. Norwood, NJ : Ablex, p.109-117.

COSTELlO, B. (1982). "The application of computerized psychological and educational assessment in South Australia », School Psychology International, 3: 195-202.

CWIKEL, J. et R. CNAAN (1991). "Ethical dilemmas in applying second-wave information technology to social work practice », Social Work, vol. 36, $\mathrm{n}^{\circ} 2: 114-120$.

ELLIS, L.B. et J.R. RAINES (1982). " Health education using microcomputers II : One year in the clinic », Preventive Medicine, II : 212-224.

ERDMAN, H.P. et S.W. FOSTER (1986). "Computer-assisted assessment with couples and families », Family Therapy, vol. 13, nº $1: 23-40$.

ERdman, H.P., Greist, J.H., Klein, M.H., Jefferson, J.W. et C. Getto (1981). « The computer psychiatrist : How far have we come ? Where are we heading ? How far dare we go ? ", Behavior Research Methods and Instrumentation, vol. 13, $\mathrm{n}^{\circ} 4: 398$.

ERDMAN, H.P., KLEIN, M.H. et J.H. GREIST (1985). "Direct patient computer interviewing », Journal of Consulting and Clinical Psychology, vol. 53, $\mathrm{n}^{\circ} 6: 760-773$.

FRANKLIN, B. et S. MCLEMORE (1967). " A scale for measuring attitudes toward student health services », Journal of Psychology, 66 : 143-147.

GANDY, J.M. et L. TEPPERMAN (1990). False Alarm : The Computerization of Eight Welfare Organizations. Waterloo : Wilfrid Laurier University Press.

GREIST, J.H. et M.H. KLEIN (1980). "Computer programs for patients clinicians and researchers in psychiatry », dans J.B. Sidowski, J.H. Johnson et T.A. Williams (eds.), Technology in Mental Health Care Delivery Systems. Norwood, NJ : Ablex, p. 161-181.

GREIST, J.H. et M.H. KLEIN (1981). « Computers in psychiatry », dans S. Arieti et H.K.H. Brodie (eds.), American Handbook of Psychiatry, vol. 7. New York : Basic Books, p. 750-777.

HART, R.R. et M.A. GOLDSTEIN (1985). "Computer-assisted psychological assessment », Computers in Human Services, vol. 1, n 3 : 69-75.

HEDL, J.J., O'NEIL, H.F. et D.M. HANSEN (1973). "Affective relations toward computer-based intelligence testing », Journal of Consulting and Clinical Psychology, $40: 217-222$. 
HeDlund, J.L., VieWEG, B.W. et D.N. Cho (1985). « Mental health computing in the 1980's : II. Clinical applications », Computers in Human Services, vol. 1, $\mathrm{n}^{\circ} 2: 1-31$.

HEDLUND, J.L. (1988). "Mental health computing : Directions for research", Computers in Human Services, vol. 2, $\mathrm{n}^{\text {os }}$ 3-4 : 9-36.

HEISE, D.R. (1965). "Semantic differential profiles for 1000 most frequent English words », Psychological Monographs, vol. 79, n $8: 1-31$.

HEISE, D.R. (1969). "Some methodological issues in semantic differential research », Psychological Bulletin, 72 : 406-422.

HEISE, D.R. (1970). "The semantic differential and attitude research », dans G. Summers (ed.) Attitude Measurement. Chicago : Rand McNally, p. 235-253.

HOFER, P.J. (1985). «Developing standards for computerized psychological testing », Computers in Human Behavior, 1 : 301-315.

HOFER, P.J. et B.F. GREEN (1985). "The challenge of competence and creativity in computerized psychological testing », Journal of Consulting and Clinical Psychology, vol. 53, $\mathrm{n}^{\circ} 6: 826-838$.

HUDSON, W., NURIUS, P. et S. ReISMAN (1988). "Computerized assessment instruments : their promise and problems », Computers in Human Services, vol. 3, $\mathrm{n}^{\text {os }}$ 1-2, The Haworth Press, p. 51-70.

HULKA, B.S., CASSEL, J.C., ZYRANSKI, S.J. et S.J. THOMPSON (1970). "Scale for the measurement of attitudes towards physicians and primary medical care », Medical Care, $8: 429-435$.

JOHNSON, J.H. et T.A. WILLIAMS (1980). "Using on-line computer technology to improve services response and decision-making effectiveness in a mental health admitting system », dans J.B. Sidowski, J.H. Johnson et T.A. Williams (eds.), Technology in Mental Health Care Delivery Systems. Norwood, NJ : Ablex, p. 237-249.

JOHNSON, J.H., GODIN, S.W. et M.L. BLOOMQUIST (1981). « Human factors engineering in computerized mental health care delivery », Behavior Research Methods and Instrumentation, vol. 13, $\mathrm{n}^{\circ} 4: 425-429$.

KLINGER, D.E., JOHNSON, J.H. et T.A. WILLIAMS (1976). "Strategies in the evaluation of an on-line computer-assisted unit for intake assessment of mental health patients ", Behavior Research Methods and Instrumentation, vol. 8, $\mathrm{n}^{\circ} 2$ : 94-100.

KLINGER, D.E. et D.A. MILLER (1977). "Process evaluation of an on-line computerassisted unit for intake assessment of mental health patients », Behavior Research Methods and Instrumentation, vol. 9, $\mathrm{n}^{\circ} 2$ : 10-116.

LANYON, R.I. et J.H. JOHNSON (1980). "Technology in mental health : a conceptual overview ", dans J.B. Sidowski, J.H. Johnson et T.A. Williams (eds.), Technology in Mental Health Care Delivery Systems. Norwood, NJ : Ablex, p. 17-24.

LEMYRE, L. (1986). Stress psychologique et appréhension cognitive. Thèse de doctorat (inédite), École de psychologie, Université Laval.

LEMYRE, L., TESSIER, R. et L. FILLION (1990). Mesure de stress psychologique : manuel d'utilisation. Behaviora.

LOCKE, H.J. et K.M. WALLACE (1959). "Short marital adjustment and prediction tests : their reliability and validity », Marriage and Family Living, vol. 21, $\mathrm{n}^{\circ} 2$ : 251-255. 
LOGIE, A.R., MADIRAZZA, J.A. et I.W. WeBSTER (1976). "Patient evaluation of a computerized questionnaire », Computers and Biomedical Research, 9: 169-176.

LUCAS, R.S. (1977). "A study of patients attitudes to computer interrogation », International Journal of Man-machine Studies, 9:69-86.

LUSHENE, R.E., O'NEIL, H.F. et T. DUNN (1974). "Equivalent of a completely computerized MMPI », Journal of Personality Assessment, 38 : 353-361.

MATHISEN, K.S. (1988). "Issues in research on clinical computers applications for mental health », Computers in Human Services, vol. 2, $\mathrm{n}^{\text {os }}$ 3-4 : 87-107.

MERRELL, K.W. (1985). "Computer use in psychometric assessment : evaluating benefits and potential problems ", Computers in Human Services, vol. 1, $\mathrm{n}^{\circ} 3: 59-67$.

MEZZICH, J.E., DOW, J.T. et G.A. COFFMAN (1982). « Developing an efficient clinical information system for a comprehensive psychiatric institute: I. Principles, design and organization », Behavior Research Methods and Instrumentation, vol. $13, \mathrm{n}^{\circ} 4: 459-463$.

MOOS, R. (1974). "Systems for the assessment and classification of human environments : The overview », dans R. Moss et P. Insel (eds.), Issues in Social Ecology ; Human Milieus. Palo Alto, CA : Mayfield, p. 5-28.

NURIUS, P. (1990). "A review of automated assessment ", Computers in Human Services, vol. 6, $\mathrm{n}^{\circ} 4: 265-281$.

OsGoOd, C.E., SuCl, G.J. et P.H. Tannenbaum (1957). The Measurement of Meaning. Illinois : The University of Illinois Press.

Poulin, M., Beaudoin, A., Béliveau, G., Lamarre, A. et F. Turgeon-KraWczuk (1991). Les effets et l'appréciation de l'utilisation d'un logiciel interactif par les parents d'enfants atteints de fibrose kystique. Rapport final, recherche subventionnée par la Fondation canadienne de fibrose kystique. Laboratoire de recherche, École de service social, Université Laval, 30 novembre, 300 pages.

SAMPSON, J.P. (1983a). "Measurement forum. Computer-assisted testing and assessment : current status and implications for the future ", Measurement and Evaluation in Guidance, vol. 15, nº 3 : 293-299.

SAMPSON, J.P. (1983b). "An integrated approach to computer implications in counseling psychology », The Counseling Psychologist, vol. 11, $\mathrm{n}^{\circ} 4$ : 65-74.

SELMI, P.M., KlEIN, M.H., GREIST, J.H., JOHNSON, J.H. et W.G. HARRIS (1982). «An investigation of computer-assisted cognitive-behavior therapy in the treatment of depression », Behavior Research Methods and Instrumentation, vol. 14, $\mathrm{n}^{\circ} 2: 181-185$

SKINNER, H.A. et B.A. ALLEN (1983). "Does the computer make a difference? Computerized versus face-to-face versus self-report assessment of alcohol, drug and tobacco use », Journal of Consulting and Clinical Psychology, vol. 51, $\mathrm{n}^{\circ} 2: 267-275$.

SKINNER, H.A., STEINHAUER, P.D. et J. SANTA-BARBARA (1983). "The family assessment measure ", Canadian Journal of Community Mental Health, vol. 2, n 2 : 91-105.

SORRELL, S.P., GRIEST, J.H. et M.H. KLEIN (1982). "Enhancement of adherence to tricyclic antidepressants by computerized supervision », Behavior Research Methods and Instrumentation, vol. 14, $\mathrm{n}^{\circ} 2$ : 176-180.

SPACE, L.G. (1981). "The computer as a psychometrician », Behavior Research Methods and Instrumentation, vol. 13, $\mathrm{n}^{\circ} 4$ : 595-606. 
STOUT, R.L. (1981). « New approaches to the design of computerized interviewing and testing systems », Behavior Research Methods and Instrumentation, vol. 13, no $4: 436-442$.

Thurstone, L.L. et E.J. Chave (1992). The Measurement of Attitude. Chicago : The University of Chicago Press.

TURGEON-KRAWCZUK, F. (1991). Un logiciel interactif en service social : effets sur les parents d'enfants atteints d'une maladie chronique. Thèse de doctorat, École de service social, Université Laval. 